\title{
Stability and response bounds of non-conservative linear systems
}

\author{
Pommer, Christian
}

Published in:

2003 International Conference Physics and Control. Proceedings.

Publication date:

2003

Document Version

Publisher's PDF, also known as Version of record

Link back to DTU Orbit

Citation (APA):

Pommer, C. (2003). Stability and response bounds of non-conservative linear systems. In 2003 International Conference Physics and Control. Proceedings. (Vol. 4, pp. 1106-1109). IEEE.

\section{General rights}

Copyright and moral rights for the publications made accessible in the public portal are retained by the authors and/or other copyright owners and it is a condition of accessing publications that users recognise and abide by the legal requirements associated with these rights.

- Users may download and print one copy of any publication from the public portal for the purpose of private study or research.

- You may not further distribute the material or use it for any profit-making activity or commercial gain

- You may freely distribute the URL identifying the publication in the public portal

If you believe that this document breaches copyright please contact us providing details, and we will remove access to the work immediately and investigate your claim 


\title{
Stability and response bounds of non-conservative linear systems
}

\author{
Christian Pommer \\ Department of Mathematics \\ Technical University of Denmark \\ DK-2800 Kgs.Lyngby \\ C.Pommer@mat.dtu.dk, ht.tp://www.mat.dtu.dk/people/C.Pommer
}

\begin{abstract}
For a linear systen of second order differential equations the stability is studied by Lyapunov's direct method. The Lyapunov matrix equation is solved and a sufficient condition for stability is expressed by the system matrices. For a system which satisfies the condition for stability the Lyapunov function is used to derive amplitude bounds of displacement and velocity in the homogeneous as well as in the inhomogeneous case. The developed results are illustrated by examples.
\end{abstract}

\section{Introduction}

Given the lincar system of differential equations of the form

$$
M \ddot{x}(t)+(D)+G) \dot{x}(t)+(K+N) x(t)=f(t)
$$

where $M, D$ and $K$ are Hermitian (in particular real symrnetric) matrices. The mass matrix $M$, the damping matrix $D$ and the stiffiness matrix $K$ are all positive definite $(M>0, D>0, K>0)$, while the matrix $G$ of the gyroscopic forces and the matrix $N$ of the circulatory forces are skew-Hermitian (in particular real skew-symmetric). If $G=0$ and $N=0$ we deal with a clissical darnped system which of course is stable. Recently response bounds of such systems are given by Schiehlen, Hu and Eberhard[1, 2, 3, 4]. If both circulatory forces $N$ and gyroscopic forces $G$ are present, the stability of the system depends on the relation botween the stabilizing forces characterized by $D$ and $G$ and the destabilizing forces characterized by $N$. If the system is stable, we can ask for bounds of the response of the system. To examine whether the system is stable, we find a Lyapunov function by solving the Lyapunov matrix equation. Then a sufficient condition for stability is expressed in ticrms of the properties of the system matrices. This stability condition includes for a certain choice of the involved parameters the more restrictive stat- bility criterion earlier found by Frik[5]. We then achieve bounds of the responses by using the Lyapunov function associated with the stable system. Finally we give exarnples which demonstrate the usefulness of the results.

\section{Lyapunov's direct method}

The homogeneous linear system obtained from (1)

$$
M \ddot{x}(t)+(D+G) \dot{x}(t)+(K+N) x(t)=0
$$

can be rewritten as a first order system $\dot{z}=A z$ where

$$
A=\left(\begin{array}{cc}
0 & I \\
-K-N & -D-G
\end{array}\right), z=\left(\begin{array}{c}
x \\
\dot{x}
\end{array}\right)
$$

and $I$ is the identity matrix. As a Lyapunov function $V(z)$ for system (3) we take

$$
V=z^{*}(t) P z(t)
$$

where $P=P^{*}$ is a Hermitian matrix, which satisfies the. Lyapunov matrix equation

$$
P A+A^{*} P=-Q
$$

and $Q=Q^{*} \geq 0$. 'The system (3) is asymptotically stable, if there exist Hermitian matrices $P>0$ and $Q>0$ which satisfy the Lyapunov matrix equation (5).

\section{Stability}

The crucial point here is to find a matrix $P$ satisfying (5). Starting with a first integral of the of the equation of motion we find a proper solution $P$. We then formulate the following

Theorem: If $b^{2}-4 a c>0$ and $b>0$, then system (2) is asymptotically stable. 
The scalars $a, b$ and $c$ are defined by

$$
\begin{array}{r}
a=\lambda_{\max }\left(M+G^{*} K^{-1} G\right), \\
b=\lambda_{\min }\left(2 D+\frac{1}{2}\left(G^{*} K^{-1} N+N^{*} K^{-1} G\right)\right), \\
c=\lambda_{\max }\left(N^{*} K^{-1} N\right),
\end{array}
$$

where $\lambda_{\min }$ and $\lambda_{\max }$ denote the smallest and the largest eigenvalues of the respective matrices. A more restrictive stability condition we achieve by making a rough estimate of the scalars $a$ and $b$

$$
\begin{gathered}
a \leq m_{\max }+\frac{1}{4} g_{\max }^{2} / k_{\min }, \\
b \geq 2 d_{\min }-g_{\max } n_{\max } / k_{\min } .
\end{gathered}
$$

Here $m_{\max }$ is the largest eigenvalue of $M, g_{\max }$ and $n_{\text {max }}$ are largest values of the moduli of the eigenvalues of $G$ and $N$, respectively, and $k_{\text {min }}$ derotes the smallest eigenvalue of $K$. Then the stability conditions of the theorem become

$$
\begin{aligned}
\left(2 d_{\min }-g_{\max } n_{\text {max }} / k_{\text {min }}\right)^{2} & \\
-4\left(m_{\max }+\frac{1}{4} g_{\max }^{2} / k_{\min }\right) n_{\max }^{2} / k_{\min } & >0, \\
2 d_{\min }-g_{\max } n_{\max } / k_{\min } & >0 .
\end{aligned}
$$

Both inequalities in (8) are satisfied if

$$
\begin{array}{r}
d_{m i n}^{2} k_{\min }-d_{\min } g_{\max } n_{\max } \\
-n_{\text {max }} n_{\text {max }}^{2}>0
\end{array}
$$

This inequality is known as a sufficient condition for asymptotic stability, se Frik[5] and Kliem and Seyranian!6] .

\section{Response bounds}

First, we consider the homogeneous system (2) which we assume to be stable according to the stability theoren. The Lyapunov function can be used to estimate the 2-norm $\|x(t)\|=\sqrt{x^{*}(t) x(t)}$ as follows

$$
\|x(t)\| \leq \sqrt{\frac{V_{0}}{\lambda_{\min }\left(K+\frac{\gamma}{2} D-\frac{\gamma^{2}}{4} M\right)}},
$$

where $\gamma=b /(2 a)$ and $V_{0}$ is the value of the Lyapunov function for $t=0$ which sounds

$$
\begin{array}{r}
V_{0}=x^{*}(0)\left(K+\frac{\gamma}{2} D-\frac{\gamma^{2}}{4} M\right) x(0)+ \\
\left(\dot{x}(0)+\frac{\gamma}{2} x(0)\right)^{*} M\left(\dot{x}(0)+\frac{\gamma}{2} x(0)\right) .
\end{array}
$$

In a similar way we obtain the estimate for the time derivativt:

$$
\|\dot{x}(t)\| \leq \frac{\gamma}{2}\|x(t)\|+\sqrt{\frac{V_{0}}{\lambda_{\min }(M)}} .
$$

We now return to the inhomogeneous system (1) which wo again assume to be stable according to the theorem. If we assume $f(t)$ to be a non-transient excitation, it is normally easy to find a particular solution $x_{\text {part }}(t)$ e.g. by making a suitable guess. Since every solution $x(t)$ to (1) can be expressed as a sum of a solution $x_{\text {hom }}(t)$ to the homogeneous equation (2) and a particular solution $x_{\text {part }}(t)$ to the inhomogeneous equation (1) we achieve the bound as follows

$$
\begin{array}{r}
\|x(t)\| \leq \sqrt{\frac{V_{0, h}}{\lambda_{\min }\left(K+\frac{\gamma}{2} D-\frac{\gamma^{2}}{4} M\right)}} \\
+\left\|x_{\text {part }}(t)\right\|,
\end{array}
$$

where $V_{0, h}$ is given by (11) if we in on the right side substitute $x(t)$ by the $x_{\text {hom }}(t)$. For a transient excitation $f(t)$ we find a solution to (1) with the given initial conditions $x(0)=0$ and $\dot{x}(0)=0$ by calculating the convolution of the impulse response matrix $\Phi(t)$ and $f(t)$

$$
x(t)=\int_{0}^{t} \Phi(t-\tau) f(\tau) d \tau .
$$

Taking $f(t)=u \psi(t)$, where $u$ is a constant vector and $\psi(t)$ is a scalar function subjected to the condition

$$
p=\int_{0}^{\infty}\|\psi(t)\| d t<\infty \quad
$$

we can deduce

$$
\|x(t)\| \leq \sqrt{\frac{u^{*} M^{-1} u}{\lambda_{\min }\left(K+\frac{\gamma}{2} D-\frac{\gamma^{2}}{4} M\right)}} p .
$$

\section{Examples}

\subsection{Example 1}

To illustrate the formulas for response bounds of the homogeneous system (2) let us consider the $3 \times 3$ system described by

$$
\begin{aligned}
& M=\left(\begin{array}{rrr}
3 & 1 & -1 \\
1 & 3 & 1 \\
-1 & 1 & 3
\end{array}\right), \\
& D=\left(\begin{array}{rrr}
8 & -2 & 2 \\
-2 & 8 & -2 \\
2 & -2 & 8
\end{array}\right) \text {, } \\
& G=\left(\begin{array}{rrr}
0 & 2 & 3 \\
-2 & 0 & 2 \\
-3 & -2 & 0
\end{array}\right) \text {, } \\
& K=\left(\begin{array}{lll}
4 & 2 & 3 \\
2 & 4 & 2 \\
3 & 2 & 4
\end{array}\right) \text {, } \\
& N=\left(\begin{array}{rrr}
0 & 1 & 1 \\
-1 & 0 & 1 \\
-1 & -1 & 0
\end{array}\right) \text {. }
\end{aligned}
$$




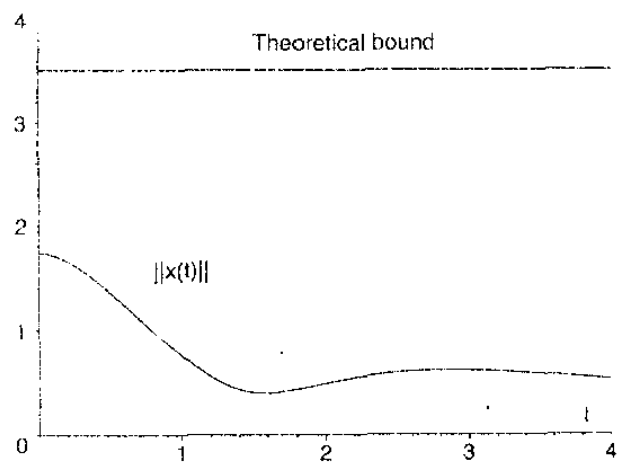

Figure 1: Theoretical bound and $\|x(t)\|$.

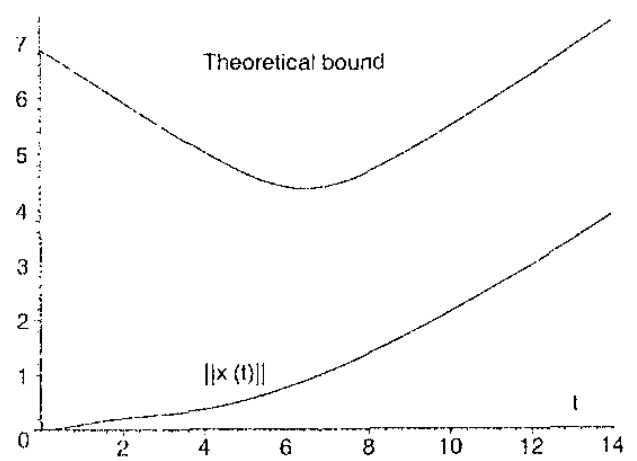

Figure 2: 'Theoretical bound and $\|x(t)\|$

Given the initial conditions $x(0)=[1,1,1]^{\mathrm{T}}$ and $\dot{x}(0)=[0,0,0]^{T}$, we calculate the constants defined in (6) as

$$
\begin{array}{r}
a=\lambda_{\max }\left(M+G^{*} K^{-1} G\right)=\frac{37+\sqrt{809}}{8}, \\
b=\lambda_{\min }\left(2 D+\frac{1}{2}\left(G^{*} K^{-1} N+N^{*} K^{-1} G\right)\right)=13, \\
c=\lambda_{\max }\left(N^{*} K^{-1} N\right)=3 .
\end{array}
$$

Because $b>0$ and $b^{2}-4 a c=70.8>0$ the system is stable according to the stability theorem, while the fik criterion given by $(9)$ is not satisfied in this case. To get the bolind of the 2-norm given by (10) we first, calculate the values $\gamma=b /(2 a)=0.794, V_{0}=33.94$ and $\lambda_{\min }\left(K+\frac{\gamma}{2} D-\frac{\gamma^{2}}{4} M\right)=2.752$. The bound is then found to be $\|x(t)\|<3.51$ and this value is compared to the exact value of $\|x(t)\|$ as shown in figure 1. Using $\lambda_{\min }(M)=1$ we can calculate the bound of $\|\dot{x}(t)\|$ according to equation (12). Figure 2 shows the bound of $\|\dot{x}(t)\|$ compared to the exact value of $\|\dot{x}(t)\|$.

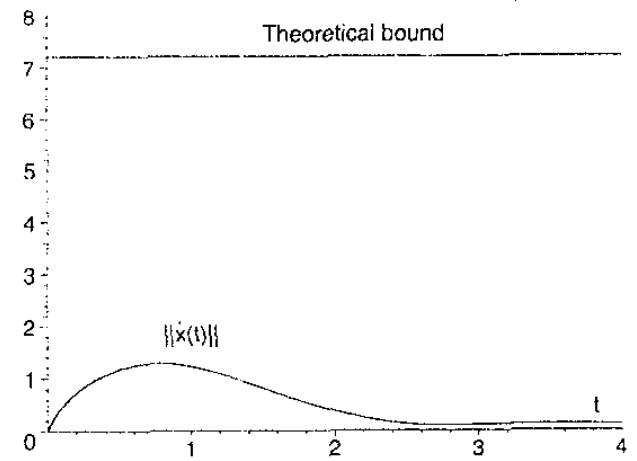

Figure 3: Theoretical bound and $\|\dot{x}(t)\|$

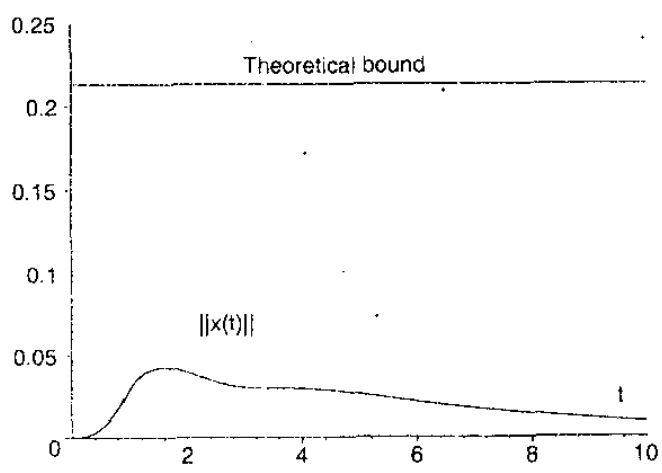

Figure 4: Theoretical bound and $\|\dot{x}(t)\|$

\subsection{Example 2}

We now look at the inhomogeneous system (1) with the same system matrices (17) as given in example 1

. We assume that the system is excited by a transient force given by $f(t)=[t, 1,1]^{T}$. A particular solution to the inhomogeneous equation is

$$
x_{p a r t}(t)=t\left(\begin{array}{c}
13 / 30 \\
1 / 15 \\
-7 / 30
\end{array}\right)+\left(\begin{array}{c}
-1087 / 450 \\
-233 / 450 \\
1003 / 450
\end{array}\right) .
$$

With the initial conditions $x(0)=[0,0,0]^{T}$ and $\dot{x}(0)=[0,0,0]^{T}$ we have for the initial conditions of $x_{\text {hom }}(t)$

$$
\begin{gathered}
x_{h o m}(0)=-\left(\begin{array}{c}
-1087 / 450 \\
-233 / 450 \\
1003 / 450
\end{array}\right), \\
\dot{x}_{h o m}(0)=-\left(\begin{array}{c}
13 / 30 \\
1 / 15 \\
-7 / 30
\end{array}\right)
\end{gathered}
$$


Substituting $x_{\text {hom }}(0)$ and $\dot{x}_{\text {hom }}(0)$ into (11) we obtain $V_{0, h}=34.69$. The bound of the 2 -norm given by $(13)$ is then found to be $\|x(t)\|<3.55+\left\|x_{\text {part }}(t)\right\|$ . This value is compared to the exact norm $\|x(t)\|$ as shown in figure 3 .

If we instead excite the system with a transient force, say $f(t):=u \psi(t)$, where $u:=[1,0,0]^{T}$ and $\psi(t)=t$ for $0<t<1$ and otherwise $\psi(t)=0$, we have $p=1 / 2$ and $u^{*} M^{-1} u=1 / 2$. Then the equation (16) gives the bound of the 2 -norm $\|x(t)\|<0.213$. This value is be compared to the exact value of the norm $\|x(t)\|$ as shown in figure 4 .

\section{Conclusions}

Using the Lyapunov's direct method we have formulated a sufficient condition for stability of a certain class of non-conservative systems. The condition is expressed by the largest and smallest eigenvalues of combinations of the system matrices. The constructed Lyapunov function is used to obtain bounds for the norms of the displacement and the velocity. There exist stable non-conservative systems, which do not satisfy the deduced condition for stability. For such systems, no response bounds are available by this method.

\section{References}

[1] W. Schiehlen and B. Hu, "Amplitude Bounds of Linear free Vibrations", ASME J. Appl. Mech. 6 (1995) 231-233.

[2] W. Schichlen and B. Hu, "Ainplitude Bounds of Linear Vibration Responses", ZAMM Z. angew. Math. Mech. 76 (1996) 453-454.

[3] B. Hu and W. Schiehlen, "Amplitude bounds of linear forced vibrations", Arch. Appl. Mech. 66 (1996) 357-368.

[4] B. Hu and P. Eberhard, "Response Bounds for Line:ar Damped Systems", ASME J. Appl. Mech. 66 (1999) 997-1003.

[5] M. Frik, "Zur Stabilität nichtkonservativer linearer Systeme", ZAMM Z. angew. Math. Mech. 52 (1972) T47-T49.

[6] W. Kliem and A. P. Seyranian, "Metelitsyn's Inequality and Stability Criteria for Mechanical Sys1.ems", to appear in J. Appl. Maths Mechs. 